BBA 45637

\title{
THE PREPARATION AND CHARACTERIZATION OF PHOSPHORYLATED DERIVATIVES OF HISTIDINE
}

\author{
D. E. HULTQUIST
}

Department of Biological Chemistry, The University of Michigan, Ann Arbor, Mich. (U.S.A.)

(Received September I4th, 1967)

\section{SUMMARY}

I. Phosphorylated derivatives of histidine were synthesized and studied in order to provide a better understanding of the chemistry of phosphohistidine-containing proteins. I-Phosphohistidine, I,3-diphosphohistidine, $\alpha$, I,3-triphosphohistidine, and phosphorylated derivatives of $\alpha-N$-acetylhistidine were prepared by reactions with $\mathrm{POCl}_{3}$ or phosphoramidate. The compounds were purified by ion-exchange chromatography and characterized by the phosphate: histidine ratio, electrophoretic behavior, and ultraviolet spectra.

2. The rates of hydrolysis of these compounds, as well as 3 -phosphohistidine, phosphoimidazole, and I,3-diphosphoimidazole, were determined over a wide range of $\mathrm{H}^{+}$concentrations. In strongly acidic solution all of the compounds appeared to be hydrolyzed by the same mechanism. In all cases the $\mathrm{N}$-phosphoryl bond was hydrolyzed more readily if a proton or phosphoryl group were substituted on the other ring nitrogen, and histidine derivatives were less stable than the corresponding imidazole derivatives.

3. For both mono- and diphosphoryl derivatives of histidine, the $\mathrm{N}$-I phosphoryl group was hydrolyzed and transferred to other imidazole compounds more readily than the N-3 phosphoryl group. The greater reactivity at the N-I position is partially due to the presence of the protonated $\alpha$-amino group.

4. In aqueous solution, I-phosphohistidine is rapidly converted to 3-phosphohistidine, histidine and inorganic phosphate.

\section{INTRODUCTION}

Phosphohistidine has been found to occur in peptide linkage in a number of phosphoproteins. 3-Phosphohistidine has been isolated from the phosphorylated form of succinate thiokinase $\mathrm{e}^{1-3}$ and it appears to be this isomer which has been isolated from a phosphopeptide of Escherichia coli ${ }^{4}$ and from a phosphorylated form of nucleoside diphosphokinase ${ }^{5}$. Evidence has recently been presented for the occurrence of I-phosphohistidine in alkaline hydrolysates of rat-liver supernatant ${ }^{6}$.

Whereas both I- and 3-phosphohistidine have been prepared chemically, the physical properties and rates of hydrolysis have been determined for only the 3 isomer ${ }^{3}$. The present paper reports the characterization of I-phosphohistidine and the prepa- 
ration, isolation, and characterization of I,3-diphosphohistidine, $\alpha$, I,3-triphosphohistidine and phosphorylated derivatives of $\alpha-N$-acetylhistidine.

This study was undertaken to provide methods for identifying I-phosphohistidine and to establish conditions which would allow phosphohistidine-containing proteins to be studied without hydrolysis or transfer of the phosphoryl group. Interest in the reactivity of the phosphorylated compounds described in this paper also arose from the realization that this information was needed in order to develop a method for selectively phosphorylating the imidazole groups of proteins.

\section{EXPERIMENTAL PROCEDURE}

\section{Materials}

Monohydrated $\alpha$-N-acetyl-L-histidine and L-histidine methyl ester $\cdot 2 \mathrm{HCl}$ were obtained from California Corp. for Biochemical Research, L-histidine from Sigma Chemical Company, and imidazole from Eastman Organic Chemicals.

Published procedures were used for the preparation of monopotassium phosphoramidate ${ }^{7}$, the calcium and sodium salts of phosphoimidazole and diphosphoimidazole ${ }^{8}$, the lithium salt of 3 -phosphohistidine ${ }^{3}$, and the calcium salt of $\alpha-N$-acetyl3-phosphohistidine ${ }^{3}$.

\section{General methods}

Analysis for $\mathrm{P}_{1}$ and histidine after acid hydrolysis, high-voltage electrophoresis at $\mathrm{pH}$ 8.25, and detection with Pauly spray of imidazole derivatives were carried out as described previously ${ }^{1,3}$. Hydrolysis of phosphoimidazoles on electrophoretograms was accomplished by spraying the paper with glacial acetic acid and heating at $100^{\circ}$ for $30 \mathrm{~min}$. The calcium salts of the phosphorylated compounds were converted to the sodium salts by stirring with the sodium form of Dowex 50 for $30 \mathrm{~min}$ at $0^{\circ}$. All samples of the phosphorylated compounds were stored at $-20^{\circ}$.

\section{Isolation of I-phosphohistidine}

I-Phosphohistidine was prepared, purified, and desalted on a scale 5 times larger than previously reported ${ }^{3}$. The calcium salt was precipitated by the following procedure carried out at $0^{\circ}$. Saturated aqueous $\mathrm{CaCl}_{2}$ was added to the I-phosphohistidine solution in $0.2-\mathrm{ml}$ quantities until no further precipitation occurred and then an additional $\mathrm{I} \mathrm{ml}$ was added. The $\mathrm{pH}$ was maintained at 9.0 with aq. KOH. After I5 min the precipitate was removed by centrifugation and discarded. The calcium salt of I-phosphohistidine was then precipitated by adding 2 vol. of ethanol. After 5 min the product was removed by centrifugation, washed with cold ethanol, and dried under vacuum at $25^{\circ}$.

\section{Preparation and purification of 1,3-diphosphohistidine and $\alpha, 1,3$-triphosphohistidine}

The di- and triphospho derivatives of histidine were prepared by the reaction of histidine with phosphoramidate at $4^{\circ}$. A solution of $0.4 \mathrm{~g}$ of histidine and $4 \mathrm{~g}$ of phosphoramidate in $\mathrm{I} 0 \mathrm{O} \mathrm{ml}$ of water was maintained at $\mathrm{pH} 8.0$ for 3 days. The reaction mixture was then diluted to $\mathrm{I} 1$, adjusted to $\mathrm{pH} 8.5$ with $\mathrm{KOH}$, and charged onto a $2.4 \mathrm{~cm} \times 30 \mathrm{~cm}$ column of Dowex I. The column was eluted at $4^{\circ}$ in a $0 . \mathrm{I} \mathrm{M}$ Tris chloride buffer of $\mathrm{pH} 8.25$ with a gradient of o to r.o M potassium bicarbonatecarbonate. $800 \mathrm{ml}$ of each eluent were employed. 
Histidine was also allowed to react with $\mathrm{POCl}_{3}$ at $\mathrm{pH}$ II and $0^{\circ}$ according to the procedure used to prepare diphosphoimidazole ${ }^{8}$. A solution of $0.5 \mathrm{~g}$ of histidine in $3.3 \mathrm{ml}$ of water was adjusted to $\mathrm{pH}$ II with conc. $\mathrm{LiOH}$. $\mathrm{POCl}_{3}(0.92 \mathrm{ml})$ was added slowly over a 2 -h period from a burette which projected into the histidine solution. The $\mathrm{pH}$ was maintained at I I by the addition of the lithium hydroxide solution and the temperature was maintained between 0 and $5^{\circ}$. After all of the $\mathrm{POCl}_{3}$ had been added, $\mathrm{pH}$ and temperature were maintained for an additional $30 \mathrm{~min}$. The reaction mixture was centrifuged and the supernatant was then diluted to I 1 and charged at $4^{\circ}$ onto a $2.4 \mathrm{~cm} \times 35 \mathrm{~cm}$ Dowex I column. The column was eluted at $\mathrm{pH}$ II and $4^{\circ}$ with a $0.05 \mathrm{M}$ to I.2 M potassium carbonate gradient. $1500 \mathrm{ml}$ of each eluent were used. The samples of di- and triphosphohistidine were desalted using perchloric acid, and adjusted to $\mathrm{pH}$ II with $\mathrm{KOH}$. In some cases these compounds were precipitated as the calcium salts by adding aqueous $\mathrm{CaCl}_{2}$ and maintaining the $\mathrm{pH}$ at $\mathrm{II}$ with $\mathrm{KOH}$.

\section{Phosphorylation of $\alpha-N$-acetylhistidine and separation of products}

$\alpha-N$-Acetylhistidine was also allowed to react with $\mathrm{POCl}_{3}$ at $o^{\circ}$ and $\mathrm{pH}$ II. $\alpha-N$-Acetyl-3-phosphohistidine and $\alpha-N$-acetyl-I,3-diphosphohistidine were isolated from the reaction mixture by the chromatographic method used to separate di- and triphosphohistidine, except that a 0.05 to I.oo M potassium carbonate gradient was used with $\mathrm{I} .2 \mathrm{I}$ of each eluent. After $\mathrm{CO}_{2}$ was liberated by the $\mathrm{HClO}_{4}$ treatment the solutions were adjusted to $\mathrm{pH}$ I I with $\mathrm{KOH}$.

\section{Hydrolysis}

Hydrolytic studies were carried out on solutions of the isolated solid salts or, in the case of I,3-diphosphohistidine and $\alpha, \mathrm{I}, 3$-triphosphohistidine, on solutions obtained by Dowex I chromatography and subsequent evolution of $\mathrm{CO}_{2}$ with $\mathrm{HClO}_{4}$. Rates of hydrolysis were determined by following the decrease in absorbance in the 228-235-m $\mu$ range $^{3}$ and by following the appearance of free histidine. For the latter method, approx. $0.5 \mu$ mole of the sodium salt of I-phosphohistidine in $0.050 \mathrm{ml}$ of water was added to $2 \mathrm{ml}$ of buffer at constant temperature. At intervals of time, o.I-ml aliquots were removed and analyzed for free histidine photometrically at $480 \mathrm{~m} \mu$ by a modification of the Pauly reaction'. Hydrolyses of $\mathrm{I}, 3$-diphospho derivatives were carried out at $\mathrm{pH}$ values where the hydrolysis of the corresponding monophospho derivative was either negligible or small. In the latter case correction was made using the rates of hydrolysis of the monophospho derivatives determined in separate experiments.

\section{Phosphoryl transfer reactions}

Reactions were carried out with sodium I-phosphohistidine, lithium 3-phosphohistidine, and sodium I,3-diphosphohistidine as phosphoryl donors, and with histidine and $\alpha-N$-acetylhistidine as phosphoryl acceptors. Reactions proceeded at $4^{\circ}$ in o.I M Tris buffer of $\mathrm{pH} 8.5$. The phosphoryl acceptor concentration was $0.25 \mathrm{M}$ and the phosphoryl donor concentration was such that the compound could be easily detected with the Pauly reagent when $5 \mu$ l of the reaction mixture were spotted on chromatography paper. The progress of the reaction was assessed by electrophoretic analysis and products were identified by co-electrophoresis with authentic compounds. 
RESULTS

\section{I-Phosphohistidine}

Electrophoretic analysis of the I-phosphohistidine shortly after it was eluted from the Dowex I column showed no 3-phosphohistidine, I,3-diphosphohistidine, or histidine. Upon standing, however, I-phosphohistidine reacted to give a mixture of these compounds. The relative amounts of products varied with the $\mathrm{pH}$ and temperature. If desalting, precipitation of the calcium salt, and conversion to the sodium salt were carried out rapidly at $4^{\circ}$ only very small amounts of histidine and 3-phosphohistidine were formed. However, all samples of sodium I-phosphohistidine were contaminated with $\mathrm{P}_{1}$. Some preparations of the solid calcium salt were stable for a week at $-20^{\circ}$ whereas other preparations showed conversion after one day. Like the other solid phosphoimidazole salts obtained in this study, the I-phosphohistidine contained considerable amounts of salts.

\section{I,3-Diphosphohistidine, $\alpha, 1,3$-triphosphohistidine, $\alpha$ - $N$-acetyl-3-phosphohistidine, and} $\alpha$-N-acetyl-I,3-diphosphohistidine

Phosphorylated derivatives of histidine, prepared by reaction of histidine with $\mathrm{POCl}_{3}$, were separated by Dowex I chromatography at pH I I as shown in Fig. I.

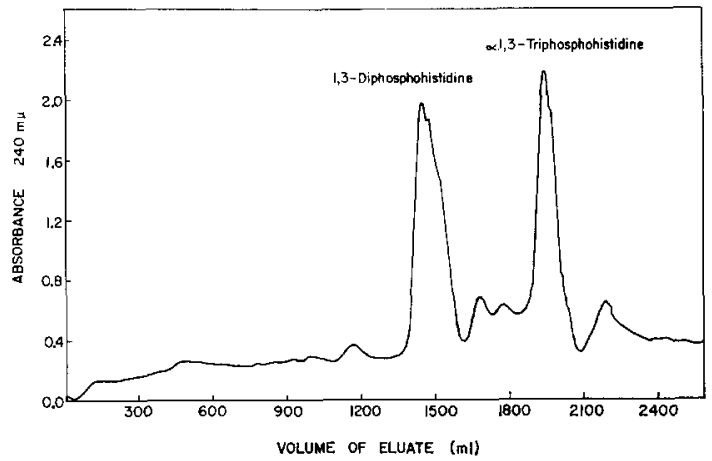

Fig. I. Chromatography on Dowex I of phosphorylated derivatives prepared by the reaction of histidine with $\mathrm{POCl}_{3}$. The column was eluted with a carbonate gradient at $\mathrm{pH}$ I $\mathrm{I}$ and $4^{\circ}$. For details SEe EXPERIMENTAI PROCEDURE.

Electrophoretic analysis showed that 1,3-diphosphohistidine which was eluted from the column could be desalted, precipitated as the calcium salt and converted to the sodium salt with little or no hydrolysis to monophosphohistidine. The $\alpha$-I,3-triphosphohistidine did not hydrolyze during the desalting procedure but during precipitation of the calcium salt and subsequent conversion to the sodium salt it was hydrolyzed to a large extent to I,3-diphosphohistidine. Fig. 2 depicts the results of electrophoresis of these compounds and the initial reaction mixture.

Phosphorylated derivatives of histidine, prepared by reaction of histidine with phosphoramidate at $\mathrm{pH}$ 8.o, were separated on Dowex I columns with a carbonatebicarbonate gradient at $\mathrm{pH} 8.25$ and $4^{\circ}$. Electrophoretic analysis of each of the three major peaks showed the first peak to be 3-phosphohistidine and the second and third peaks to correspond to the di- and triphosphohistidine prepared by reaction of 

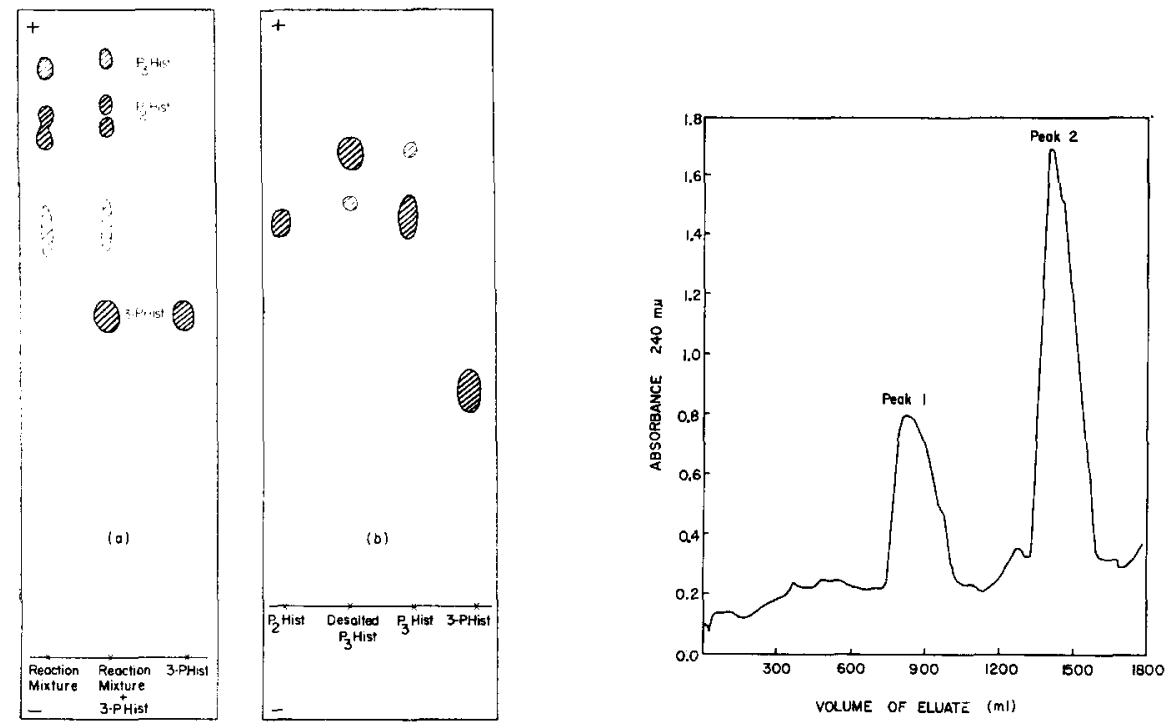

Fig. 2. Electrophoresis of $\mathrm{r}, 3$-diphosphohistidine and $\alpha, \mathrm{I}, 3$-triphosphohistidine. Compounds were detected with the Pauly reagent after acid treatment of the electrophoretograms. a. $45 \mathrm{~V} / \mathrm{cm}$ were

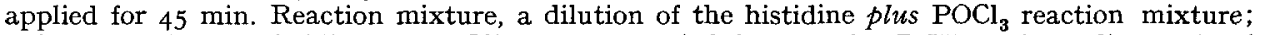
3-PHist, 3-phosphohistidine. b. $45 \mathrm{~V} / \mathrm{cm}$ were applied for $30 \mathrm{~min}$. $\mathrm{P}_{2} \mathrm{Hist}$, the sodium salt of I,3-diphosphohistidine prepared from the solid calcium salt; desalted $P_{3} H$ ist, sample of $\alpha, I, 3^{-}$ triphosphohistidine, eluted from the Dowex I column, from which $\mathrm{CO}_{2}$ has been evolved; $\mathrm{P}_{3} \mathrm{Hist}$, the sodium salt of $\alpha, 1,3$-triphosphohistidine prepared from the solid calcium salt.

Fig. 3. Chromatography on Dowex $\mathrm{r}$ of phosphorylated derivatives of $\alpha-N$-acetylhistidine prepared by reaction of $\alpha-N$-acetylhistidine with $\mathrm{POCl}_{3}$. The column was eluted with a carbonate gradient at $\mathrm{pH}$ I I and $4^{\circ}$. Identification of peaks is based on subsequent data. For details see EXPERIMENTAL PROCEDURE.

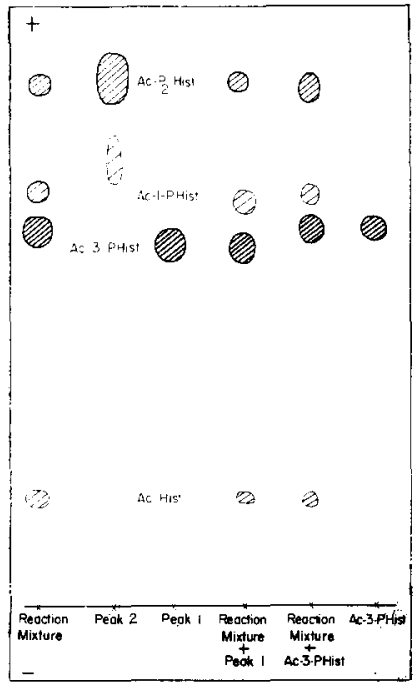

Fig. 4. Electrophoresis of phosphorylated derivatives of $\alpha$ - $N$-acetylhistidine. $45 \mathrm{~V} / \mathrm{cm}$ were applied for $36 \mathrm{~min}$. Compounds were detected with the Pauly reagent after acid treatment of the paper. Reaction mixture, a dilution of the $\alpha-N$-acetylhistidine plus $\mathrm{POCl}_{3}$ reaction mixture; Peaks $\mathrm{I}$ and 2, the major peaks eluted from the Dowex I column; Ac-3-PHist, $\alpha-N$-acetyl-3-phosphohistidine prepared by acetylation of 3 -phosphohistidine. 
histidine with $\mathrm{POCl}_{3}$. The second and third major peaks showed, after acid hydrolysis, phosphate to histidine ratios of 2.I4 and 2.99, respectively. These samples were not suitable for study because of their rapid hydrolysis in the $\mathrm{pH} 8.25$ buffer.

Chromatography of phosphorylated derivatives of $\alpha-N$-acetylhistidine, prepared by reaction with $\mathrm{POCl}_{3}$, is shown in Fig. 3. Fig. 4 shows the results of electrophoresis of the column eluates, the initial reaction mixture, and the product obtained by acetylation of purified 3-phosphohistidine.

\section{Ultraviolet spectra}

The absorption maxima in the ultraviolet region of a number of imidazole derivatives are shown in Table I. The spectra of I-phosphohistidine were very similar to those previously obtained for 3-phosphohistidine. For all compounds the absorption peaks were broader at $\mathrm{pH} 7$ than at $\mathrm{pH}$ 2.I.

TABLE I

ABSORPTION MAXIMA OF PHOSPHOIMIDAZOLE COMPOUNDS

\begin{tabular}{|c|c|c|}
\hline \multirow[t]{2}{*}{ Compound } & \multicolumn{2}{|c|}{ Absorption maxima $(m \mu)$} \\
\hline & $H C l(p H 2 . I)$ & $H_{2} O\left(p H_{7}\right)$ \\
\hline Imidazole & 207 & 207 \\
\hline Phosphoimidazole $\left(\mathrm{Na}^{+}\right)$ & 210 & 210 \\
\hline I,3-Diphosphoimidazole $\left(\mathrm{Na}^{+}, \mathrm{Ca}^{2+}\right)$ & $2 I_{4}$ & 213 \\
\hline Histidine & 2 I I & 206 \\
\hline I-Phosphohistidine $\left(\mathrm{K}^{+}\right)$ & $2 \mathrm{I}_{4}^{\star}$ & 2 I 3 \\
\hline 3-Phosphohistidine $\left(\mathrm{Li}^{+}\right)$ & 215 & 2 II \\
\hline I,3-Diphosphohistidine $\left(\mathrm{Na}^{+}\right)$ & $217^{\star}$ & $2 \mathrm{I} 7$ \\
\hline$\alpha-N$-Acetylhistidine & 2 I I & $-\cdots$ \\
\hline$\alpha-N$-Acetyl-3-phosphohistidine $\left(\mathbf{K}^{+}\right)$ & $2 \times 6^{\star}$ & $\longrightarrow$ \\
\hline$\alpha-N$-Acetyl-I,3-diphosphohistidine $\left(\mathrm{K}^{+}\right)$ & $218^{\star}$ & - \\
\hline
\end{tabular}

* Spectra observed rapidly at $8^{\circ}$ to minimize hydrolysis. All other spectra observed at $25^{\circ}$.

Rates of hydrolysis

When low concentrations of phosphoimidazole compounds were employed, hydrolysis followed kinetics which were first order with respect to the phosphoimidazole compounds. Fig. 5a shows the hydrolysis of I-phosphohistidine in I.o M $\mathrm{HCl}$ at $49^{\circ}$ as measured by following the decrease in absorbance at $230 \mathrm{~m} \mu$. Fig. $5 \mathrm{~b}$ shows the hydrolysis of I-phosphohistidine at $\mathrm{pH} 5.00$ and $46^{\circ}$ as measured by the appearance of free histidine.

Some first-order rate constants for hydrolysis of I-phosphohistidine, 3-phosphohistidine, phosphoimidazole and I,3-diphosphoimidazole in acidic solution are shown in Table II. The log of these first-order rate constants are plotted versus $\log \mathrm{H}^{+}$ concentration in Fig. 6.

The first-order rate constants for the hydrolysis of I-phosphohistidine at $46^{\circ}$ are shown in Fig. 7 for the $\mathrm{pH}$ range 2 to II. Three different buffers were used in these studies. The rates of hydrolysis were followed both by the decrease in absorbance in the $230-\mathrm{m} \mu$ region and by appearance of free histidine. Also in this figure are shown the first-order rate constants for 3 -phosphohistidine for the $\mathrm{pH}$ range 2 to 5 . 
In Fig. 8 are shown the rate constants for hydrolysis at $46.5^{\circ}$ of I,3-diphosphoimidazole in acidic solution and I,3-diphosphohistidine in solutions ranging from $\mathrm{pH} 5.9$ to I0.2.

In contrast to the other compounds studied, $\alpha, I, 3$-triphosphohistidine did not hydrolyze according to first-order kinetics. The rate of hydrolysis at $46.5^{\circ}$ increased
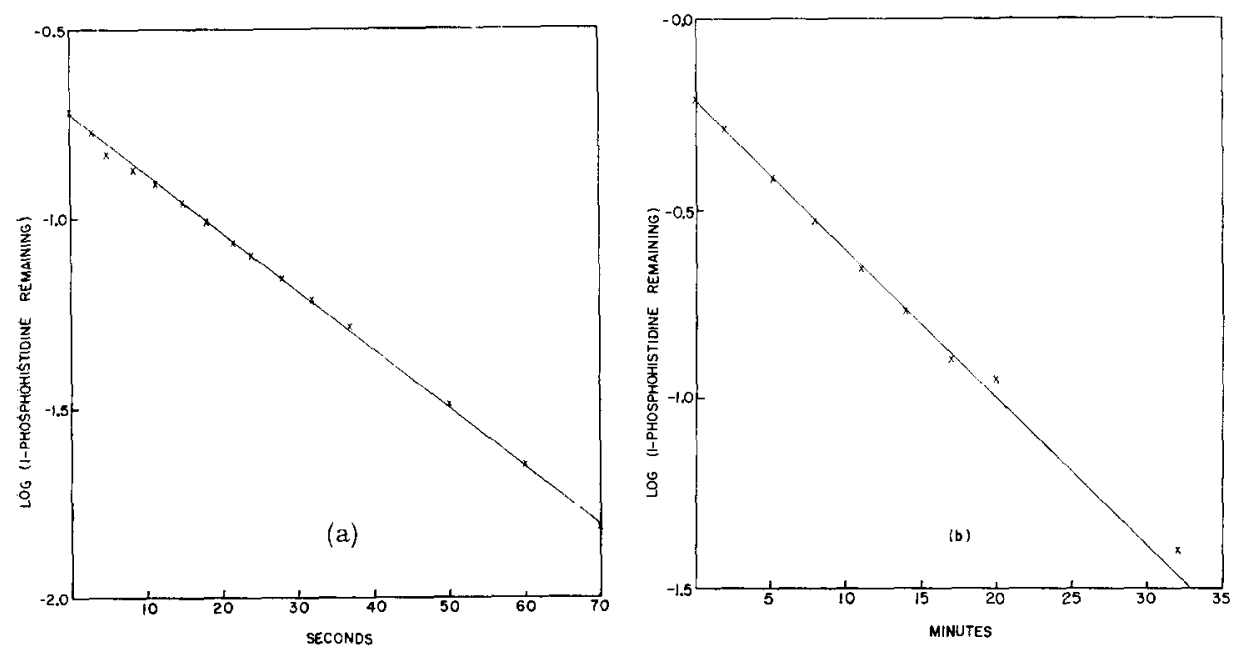

Fig. 5. Hydrolysis of I-phosphohistidine. a. The calcium salt of I-phosphohistidine was hydrolyzed in I.o $\mathrm{M} \mathrm{HCl}$ at $49^{\circ}$. Decrease in absorbance at $230 \mathrm{~m} \mu$ was followed. Absorbance at a given time minus final absorbance is a measure of I-phosphohistidine remaining at that time. The log of this quantity has been plotted versus time. b. The sodium salt of $\mathrm{I}$-phosphohistidine was hydrolyzed in $0.05 \mathrm{M}$ phosphate-0.05 $\mathrm{M}$ citrate-0.05 $\mathrm{M}$ borate at $\mathrm{pH} 5.00$ and $46^{\circ}$. Free histidine was measured photometrically. Final absorbance minus absorbance at a given time is a measure of I-phosphohistidine remaining at that time, and the log of this quantity has been plotted versus time.

TABLE II

RATE CONSTANTS $k_{1}$ FOR THE HYDROLYSIS OF PHOSPHOIMIDAZOLE COMPOUNDS IN AQ. HCl

Hydrolyses were studied by following the decrease in absorbance as described in EXPERIMENTAL PROCEDURE.

\begin{tabular}{|c|c|c|c|c|}
\hline \multirow{2}{*}{$\begin{array}{l}{[\mathrm{HCl}]} \\
(e q u i v . / l)\end{array}$} & \multicolumn{4}{|l|}{$k_{1}\left(\min ^{-1}\right)$} \\
\hline & $\begin{array}{l}\text { I-Phospho- } \\
\text { histidine }\end{array}$ & $\begin{array}{l}\text { 3-Phospho- } \\
\text { histidine }\end{array}$ & $\begin{array}{l}\text { Phospho- } \\
\text { imidazole }\end{array}$ & $\begin{array}{l}I, 3 \text {-Diphospho } \\
\text { imidazole }\end{array}$ \\
\hline $0.008 \$ \S$ & & 0.066 & o.oI65 & \\
\hline $0.032 \S \S$ & & 0.130 & 0.042 & \\
\hline O.I & & 0.31 & 0.082 & I.OO \\
\hline 0.2 & 0.87 & $0.4 \mathrm{I}$ & 0.155 & I. 76 \\
\hline 0.5 & I. 31 & 0.86 & 0.39 & $4 \cdot I$ \\
\hline I.O & 2.3 & I. 7 & 0.82 & 6.7 \\
\hline 2.0 & 3.7 & $3 \cdot 7$ & I. 8 & I 8 \\
\hline 4.0 & 7.7 & & & \\
\hline 6.0 & I2 & I6 & 9.6 & \\
\hline
\end{tabular}

* $49^{\circ}$.

$\star \star 48.5^{\circ}$.

$45.5^{\circ}$.

$\$ 46.5^{\circ}$.

$\$ \S$ Determined with a glass electrode. 
with time of incubation until it reached a value similar to the rate measured for the hydrolysis of I,3-diphosphohistidine under those conditions. This later phase of hydrolysis followed first-order kinetics. The rate at which $\alpha, 1,3$-triphosphohistidine attained the hydrolytic properties of $\mathrm{I}, 3$-diphosphohistidine steadily increased as the $\mathrm{pH}$ was lowered from 8.9 to 4.3 . At each $\mathrm{pH}$ the rate of this conversion was more rapid than the hydrolysis of any of the other compounds studied.

Electrophoretic analysis showed that dilute solutions of I-phosphohistidine and 1,3-diphosphohistidine underwent little or no hydrolysis when heated for $x_{5} \mathrm{~min}$ at $\mathrm{roo}^{\circ}$ in $0.67 \mathrm{M} \mathrm{KOH}$.

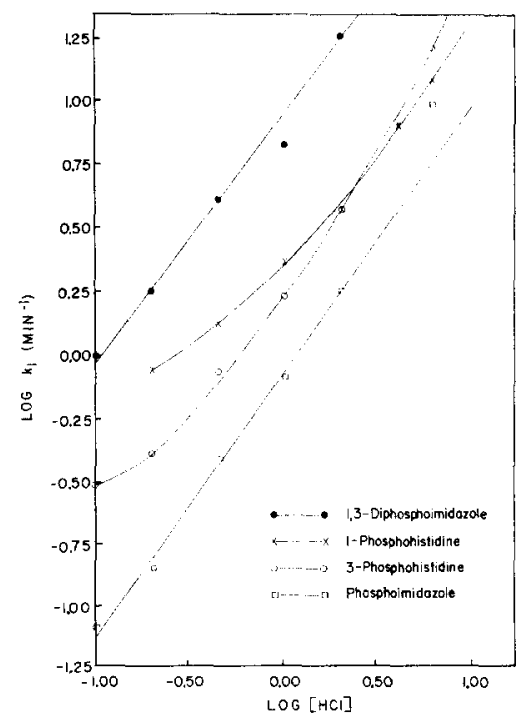

Fig. 6. Effect of $\log$ [HCl] on $\log k_{1}$ for hydrolysis of phosphoimidazole compounds. Data and details of hydrolysis are found in Table II.

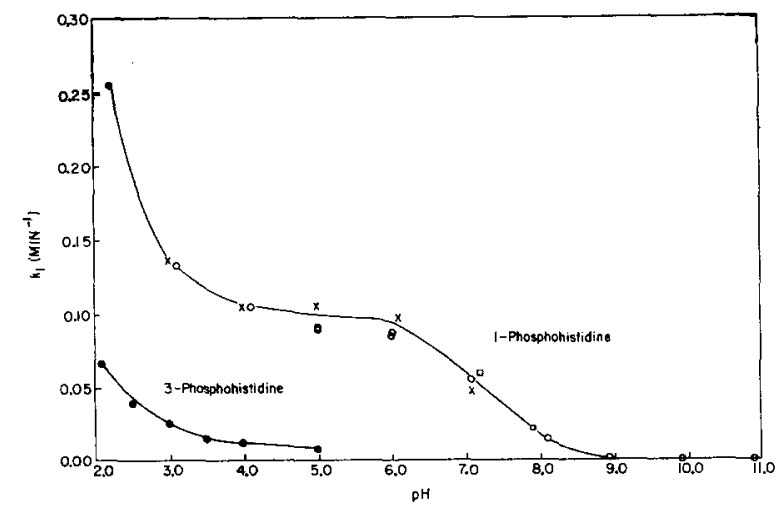

Fig. 7. Effect of $\mathrm{pH}$ on rate constant, $k_{1}$, for hydrolysis of I-phosphohistidine and 3-phosphohistidine at $46^{\circ}$. O-O, rates of hydrolysis of $\mathrm{I}$-phosphohistidine in $0.05 \mathrm{M}$ phosphate-0.05 M citrate $-0.05 \mathrm{M}$ borate buffers as determined from appearance of free histidine; $x-x$, in $0.05 \mathrm{M}$ citrate-0.05 M phosphate buffers as determined from decrease in absorbance at wavelengths ranging from 228 to $234 \mathrm{~m} \mu$; $\square-\square$, in $0.05 \mathrm{M}$ borate as determined from decrease in absorbance at $228 \mathrm{~m} \mu$; - 3-phosphohistidine in $0.05 \mathrm{M}$ citrate-0.05 $\mathrm{M}$ phosphate buffers as determined by decrease in absorbance at $233 \mathrm{~m} \mu$. 

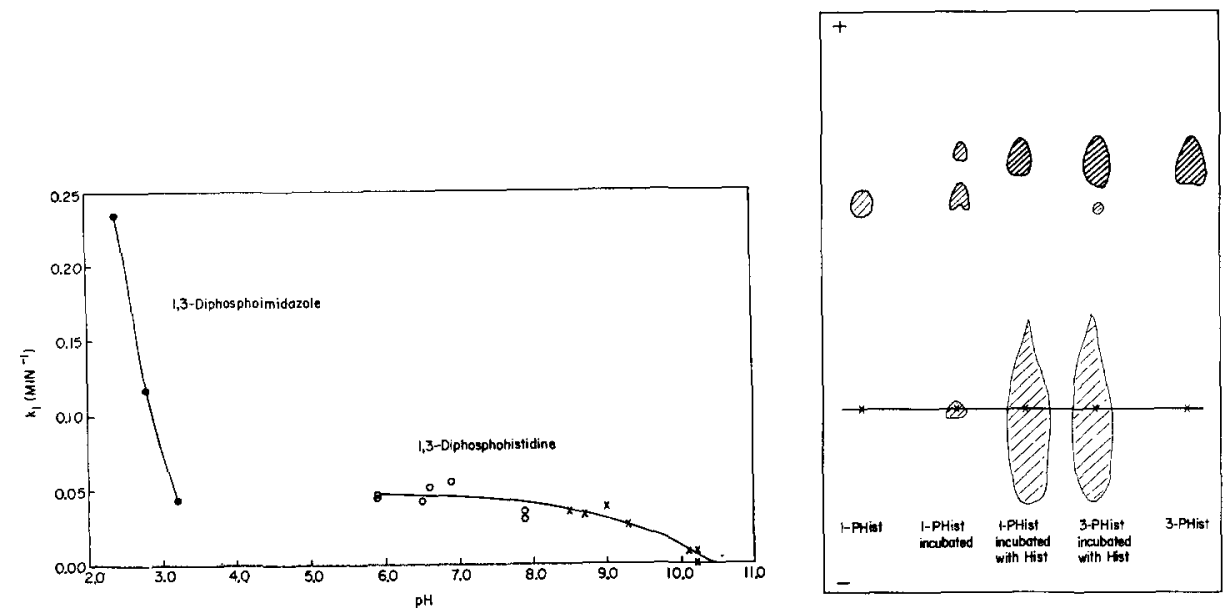

Fig. 8. Effect of $\mathrm{pH}$ on rate constant, $k_{1}$, for hydrolysis of $\mathrm{I}, 3$-diphosphoimidazole and 1,3 -diphosphohistidine at $46.5^{\circ}$. The rates of hydrolysis were followed by the decrease in absorbance at $228 \mathrm{~m} \mu$. - - $\mathrm{I}, 3$-diphosphoimidazole in $0.05 \mathrm{M}$ citrate-0.05 $\mathrm{M}$ phosphate buffers; $\mathrm{O}-\mathrm{O}$, I,3-diphosphohistidine in $0.05 \mathrm{M}$ phosphate-0.05 M borate buffers; $X-X, \mathrm{I}, 3$-diphosphohistidine in $0.05 \mathrm{M}$ borate buffers.

Fig. 9. Electrophoresis of products resulting from incubation of I-phosphohistidine, I-phosphohistidine with histidine, and 3-phosphohistidine with histidine. Histidine derivatives were detected with Pauly reagent after acid treatment of the paper. Reaction proceeded for $\mathrm{r} 2 \mathrm{~h}$ under conditions described in EXPERIMENTAL PROCEDURE. Electrophoresis was carried out for $0.6 \mathrm{~h}$ at $45 \mathrm{~V} / \mathrm{cm}$. I-PHist, I-phosphohistidine; 3-PHist, 3-phosphohistidine; Hist, histidine.

\section{Phosphoryl transfer reactions}

Paper-electrophoretic analysis showed that the following reactions proceeded at $\mathrm{pH} 8.5$ and $4^{\circ}$.

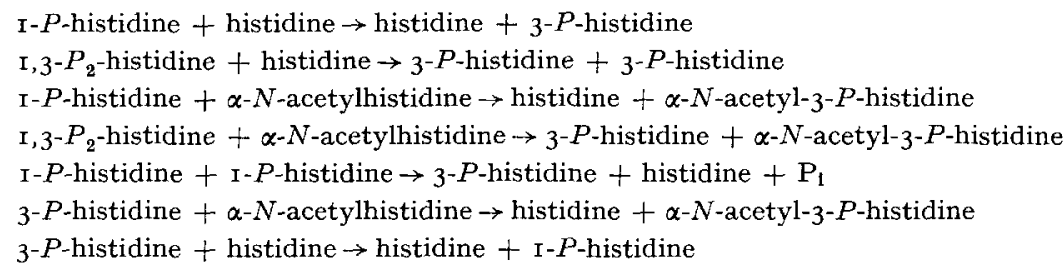

Reactions I and 2 were complete within I 2 h. After 72 h of reaction, 3 and 4 appeared to be complete, 5 was approx. $50 \%$ complete, the products of 6 were easily detectable, but the product of 7 , I-phosphohistidine, was barely detectable. Under these conditions no I-phosphohistidine was detected when 3-phosphohistidine was incubated without histidine. The electrophoretogram depicted in Fig. 9 shows the extent of reaction after I $2 \mathrm{~h}$ for Reactions I, 5 and 7 . At higher concentrations of I-phosphohistidine Reaction 5 proceeded at a faster rate.

\section{DISCUSSION}

The results of this investigation show that all three nitrogen atoms of histidine are readily phosphorylated on reaction with phosphoramidate. The formation of 
diphosphoimidazole on reaction of imidazole with phosphoramidate had previously been reported but attempts to detect a reaction of phosphoramidate with the amino group of amino acids had failed ${ }^{9}$. However, incubation of glycine or glycylglycine with phosphoramidate had been shown to result in the release of ammonia ${ }^{10}$. The present study shows that $\alpha$, I,3-triphosphohistidine can not only be formed but that it can be purified without hydrolysis of the $\alpha-N$-phospho bond. Similarly, we have been able to prepare the $\alpha-N$-phospho derivatives of many amino acids and several peptides using phosphoramidate as the phosphorylating agent.

When histidine reacts with phosphoramidate at room temperature the di- and triphospho derivatives of histidine fail to accumulate because of the rapid rates of hydrolysis and of phosphoryl transfer to other histidine compounds. At $4^{\circ}$, however, appreciable amounts of these compounds accumulate. Higher $\mathrm{pH}$ values would stabilize these products to a greater degree, but phosphorylation proceeds readily only when the amino group of phosphoramidate ( $\mathrm{p} K^{\prime}$ of 8.0$)$ is in the protonated form ${ }^{10}$.

The reaction of histidine with $\mathrm{POCl}_{3}$ at $\mathrm{pH}$ i I proceeds readily to give large amounts of di- and triphosphohistidine and only very small amounts of monophosphohistidine and histidine (Fig. I). In addition, two minor 240 -m $\mu$-absorbing compounds were eluted from the Dowex column after I,3-diphosphohistidine and before $\alpha$, I,3-triphosphohistidine. Both compounds gave a color with the Pauly reagent after acid hydrolysis. It is possible that these compounds are $\alpha, \mathrm{I}-$ and $\alpha, 3-$ diphosphohistidine. Electrophoresis of the reaction mixture shows two spots in the diphosphohistidine region (Fig. 2).

In contrast, I-phosphohistidine could not be obtained in good yield by reaction of histidine with $\mathrm{POCl}_{3}$ at $\mathrm{pH}$ II or with phosphoramidate over a range of $\mathrm{pH}$ values, temperatures, times of reaction, and concentrations of histidine and phosphoramidate. The amount of I-phosphohistidine formed by reaction with phosphoramidate goes through a maximum which, at $25^{\circ}$, is reached after approx. I $h$. The reactions responsible for the disappearance of the I-phosphohistidine are hydrolysis, phosphoryl transfer to imidazole compounds, and further reaction with phosphoramidate to yield I,3-diphosphohistidine. The phosphoryl group of I-phosphohistidine is readily transferred to the $\mathrm{N}-3$ of the imidazole rings of histidine, $\alpha-N$-acetylhistidine, and I-phosphohistidine itself. Under the conditions of phosphorylation with phosphoramidate, the 1,3 -diphosphohistidine formed undergoes reaction with water or histidine with preferential transfer of the N-I phosphoryl group. Hydrolysis yields 3-phosphohistidine and inorganic phosphate. Reaction with histidine yields two molecules of 3 -phosphohistidine. Taken together, these reactions result in a conversion of I-phosphohistidine to 3 -phosphohistidine, histidine, and inorganic phosphate.

The patterns of phosphorylation of histidine methyl ester, $\alpha-N$-acetylhistidine, and $\alpha-N$-acetylhistamine are similar to that observed with histidine. With each of these compounds short reaction times yield both the I- and 3-monophospho derivatives but one of these isomers disappears after longer times of phosphorylation. We have shown that for histidine and $\alpha-N$-acetylhistidine the monophospho derivative that accumulates after long times of reaction is the 3 isomer.

Di- and triphosphohistidine failed to separate on Dowex I columns at $\mathrm{pH} \mathrm{8.9,}$ but at $\mathrm{pH}$ is the separation was achieved and electrophoretic analysis shortly after elution revealed no hydrolysis. After I day in aq. solution at $4^{\circ}$, however, I,3-di-, 
$\alpha, \mathrm{I}, 3$-tri-, and $\alpha-N$-acetyl-r,3-diphosphohistidine showed appreciable loss of phosphate. The rates of hydrolysis and transphosphorylation are variable for the solid salts of these compounds at $-20^{\circ}$ but generally no reaction is observable after I day.

The evidence that provided the basis for the identification of $I$ - and 3-phosphohistidine and I,3-diphosphohistidine was summarized earlier ${ }^{3}$. In the present study, acid hydrolysis of diphosphohistidine released 2.14 phosphate groups per histidine residue. When diphosphohistidine was hydrolyzed under mild conditions or reacted with other imidazole compounds, 3-phosphohistidine was formed.

The properties of triphosphohistidine strongly indicate it to be the $\alpha, 1,3$-derivative rather than a derivative with an acylphosphate or pyrophosphate bond. Acid hydrolysis of this compound yields $2.99 \mathrm{P}_{1}$ groups per histidine. The extreme lability of this compound is in keeping with the lability of $\alpha-N$-phosphoryl derivatives. Mild hydrolysis yields a product which cannot be distinguished from I,3-diphosphohistidine by electrophoretic mobility or by rate of hydrolysis. Moreover, the conversion of tri- to diphosphohistidine appears to proceed without an appreciable change in the absorption at $23^{\circ} \mathrm{m} \mu$. Studies with $\alpha-N$-phospho amino acids and peptides show that hydrolysis of these compounds results in little change in absorbance at $230 \mathrm{~m} \mu$.

The three products obtained on phosphorylation of $\alpha-N$-acetylhistidine appear to be, in decreasing order of electrophoretic mobility at $\mathrm{pH} 8.25, \alpha-N$-acetyl-I,3-di-, $\alpha$ - $N$-acetyl-I-, and $\alpha$ - $N$-acetyl-3-phosphohistidine. The $\alpha$ - $N$-acetyl-3-phosphohistidine was identified by electrophoretic comparison with the authentic compound prepared by acetylating 3-phosphohistidine. The compound that migrated slightly faster than the 3-phospho derivative has been designated $\alpha-N$-acetyl-I-phosphohistidine since it, like I-phosphohistidine, disappears on longer times of phosphorylation. Identification of $\alpha-N$-acetyl-I,3-diphosphohistidine is based on the findings that, relative to $\alpha-N$-acetyl-3-phosphohistidine, this compound is more negatively charged, is more labile, and has an absorption maximum at longer wavelengths. Moreover, the compound is degraded to $\alpha-N$-acetyl-3-phosphohistidine on mild hydrolysis.

In high concentrations of acid all of the phosphoimidazole compounds studied appear to hydrolyze by the same mechanism. The straight lines with slopes of I.o, obtained by plotting $\log k_{1}$ versus $\log \mathrm{H}^{+}$concentration (Fig. 6), indicate that the hydrolysis involves a reaction between one proton and the completely protonated form of the compound.

At $\mathrm{H}^{+}$concentrations of less than $0 . \mathrm{I} \mathrm{M}$ the rates of hydrolysis of the various phosphoimidazole derivatives show markedly different $\mathrm{pH}$ dependencies. As seen from Fig. 7 , the $k_{1}$ for the hydrolysis of I-phosphohistidine is relatively constant between $\mathrm{pH}_{4}$ and 6 and then decreases sharply above $\mathrm{pH}$. These results are best explained in terms of the titration of an imidazole group with a $\mathrm{p} K^{\prime}$ of 7.3 at $46^{\circ}$. According to this hypothesis the protonated species is hydrolyzed rapidly whereas the unprotonated species is stable at $46^{\circ}$. The dependency on $\mathrm{pH}$ of the hydrolysis of 3-phosphohistidine $\mathrm{e}^{3}$ can likewise be explained in terms of the protonation of the imidazole group. Since the imidazolium $\mathrm{p} K^{\prime}$ of 3 -phosphohistidine is lower than that of $I$-phosphohistidine, the plateau is less pronounced with the 3 isomer. In contrast to the monophosphohistidines, the rate of hydrolysis of 1,3 -diphosphohistidine shows no marked change as the $\mathrm{pH}$ is increased from 5.9 to 8.5 . This is the expected behavior of a compound which has no unsubstituted imidazole nitrogen. 
I-Phosphohistidine is hydrolyzed more rapidly than $\mathrm{I}, 3$-diphosphohistidine at $\mathrm{pH} 6$ but more slowly at $\mathrm{pH}$. These results show that the $\mathrm{N}-\mathrm{I}$ phosphoryl bond is labilized considerably when a phosphoryl group is placed on $\mathrm{N}-3$ but to an even greater extent when a proton is placed on that atom. The side chain of histidine labilizes the $\mathrm{N}-\mathrm{P}$ bonds over the entire $\mathrm{pH}$ range; monophosphohistidines are hydrolyzed faster than monophosphoimidazole and I,3-diphosphohistidine faster than I,3-diphosphoimidazole.

At all $\mathrm{pH}$ values above zero the $\mathrm{N}$-I phosphoryl bond is more labile than the $\mathrm{N}-3$ phosphoryl bond. This is true when I-phosphohistidine is compared to 3-phosphohistidine and also when the two phosphoryl groups of I,3-diphosphohistidine are compared. The phosphoryl group on N-I of a mono- or diphosphohistidine is also more readily transferred to imidazole derivatives than is the corresponding $\mathrm{N}-3$ phosphoryl group. Two results suggest that the protonated $\alpha$-amino group is responsible for the lability at $\mathrm{N}$-I. The plot of $k_{1}$ versus $\mathrm{pH}$ for I,3-diphosphohistidine suggests that the decrease in rate of hydrolysis above $\mathrm{pH} 9$ corresponds to the loss of a proton from an $\alpha$-amino group with a $\mathrm{p} K^{\prime}$ of approx. 9.6. Secondly, the $\mathrm{pH}$-dependent lag in the hydrolysis of $\alpha, \mathrm{I}, 3$-triphosphohistidine suggests that the presence of a phosphoryl group on the $\alpha$-amino group stabilizes the N-I phosphoryl bond.

These studies limit the conditions that should be employed in isolating, degrading, and characterizing proteins that contain phosphoimidazole groups, especially those containing I-phosphohistidine. In order to keep I-phosphohistidine intact all procedures must be carried out in alkaline solution and in the absence of nitrogen compounds that can readily react with I-phosphohistidine. At all times the I-phosphohistidine should be in dilute solution in order to prevent its conversion to 3-phosphohistidine. The ease of non-enzymic transfer to and from imidazole groups urges one to be cautious in the interpretation of studies of biological material in which an isomer of phosphohistidine is detected or in which phosphohistidine is reported to be absent.

\section{ACKNOWLEDGEMENTS}

This investigation was supported in part by Grant 09250 from the Institute of Arthritis and Metabolic Diseases, National Institutes of Health, U.S. Public Health Service.

The author expresses thanks to Mr. Walter Andrews and Miss Joanne KaO for valuable technical assistance.

\section{REFERENCES}

i M. Deluca, K. E. Ebner, D. E. Hultquist, G. Kreil, J. B. Peter, R. W. Moyer and P. D. BoYer, Biochem. Z., 338 (I963) 5I2.

2 G. Kreil and P. D. Boyer, Biochem. Biophys. Res. Commun., I6 (I964) 55 I.

3 D. E. Hultquist, R. W. Moyer and P. D. Boyer, Biochemistry, 5 (Ig66) 322.

4 W. Kundig, S. Ghosh and S. Roseman, Proc. Natl. Acad. Sci. U.S., 52 (1964) 1067.

5 A. W. Norman, R. T. Wedding and M. K. Black, Biochem. Biophys. Res. Commun., 20 (I965) 703 .

6 O. Zetrerquist, Biochim. Biophys. Acta, 136 (I967) 279.

7 H. N. STokes, Am. Chem. J., I5 (1893) I98.

8 T. Rosenberg, Arch. Biochem. Biophys., I05 (I964) 3 I 5.

9 T. Rathlev and T. Rosenberg, Arch. Biochem. Biophys., 65 (I956) 3 I9.

io W. P. Jencks and M. Gilchrist, J. Am. Chem. Soc., 87 (I965) 3 I99. 